\title{
Control Method at Obstacle Avoidance Stage for Spacecraft
}

\author{
Xueyuan Zhang \\ School of Electrical and Electronic \\ Engineering,NCEPU,Baoding071000, Hebei, China
}

\begin{abstract}
Based on the operation data and basic parameters of spacecraft, soft landing process is done reasonable simplification equivalent. Without losing the accuracy, this paper proposes the control scheme at obstacle avoidance stage. The landing preparation track is accord with the energy conservation and the Kepler's motion law. The control strategy of soft landing can use differential to solve the optimal control strategy using Newton classical mechanics law. It has a certain practical application value in the spacecraft landing parameters calculation and track design.
\end{abstract}

Keywords-Linear discrete, Iteration method, Genetic algorithm, Gray matrix. Matlab

\section{INTRODUCTION}

With the development of aerospace industry, China's lunar exploration program has made remarkable achievements. Lunar spacecraft landing is the most important part in the lunar exploration process [1]The existing landing way has hard landing and soft landing. Soft landing is gradually reducing the landing speed through machine control, so that the spacecraft vertical velocity decreases to a very small in contact with earth or other planets surface, finally falls to the ground or other objects on the surface without damage, so as to realize the safe landing technology $[2,3]$. Based on the engineering design and practical observations available information, this paper studies the optimal control strategy in the process of lunar spacecraft soft landing.

\section{Parameters Determination In the PeRILUne AND APOLUNE}

\section{A Determine the Velocity in the Perilune and Apolune}

Assume that the airship has no energy loss in the elliptic landing orbiting, namely the total energy is conserved [4]. The total energy of the spacecraft consists of two parts: potential energy and kinetic energy. The moon surface is set as zero potential energy. Then the airship potential energy is $-m \frac{\mu}{r}$ from the moon surface $r$, and the kinetic energy is $\frac{1}{2} m v^{2}$, so the total energy is $E=\frac{1}{2} m v^{2}-m \frac{\mu}{r}$. By the energy conservation, it can get:

$$
\frac{1}{2} m v_{1}^{2}-m \frac{\mu}{r_{1}}=\frac{1}{2} m v_{2}^{2}-m \frac{\mu}{r_{2}} \text {. }
$$

By Kepler's second law, it can get $v_{1} r_{1} \sin \left(v_{1}, r_{1}\right)=v_{2} r_{2} \sin \left(v_{2}, r_{2}\right)$. As the velocity in the perilune and apolune is vertical to sagittal, so the above formula can be changed to:

$$
v_{1} r_{1}=v_{2} r_{2} \text {. }
$$

Put the formula (2) into (1), so the velocity in the perilune and apolune are $v_{1}=\sqrt{\frac{2 \mu r_{2}}{r_{1}\left(r_{1}+r_{2}\right)}}, v_{2}=\sqrt{\frac{2 \mu r_{1}}{r_{2}\left(r_{1}+r_{2}\right)}}$. As $r_{1}=1737.013+15 \mathrm{~km}=1752.013 \mathrm{~km}$,

$r_{2}=1737.013+100 \mathrm{~km}=1752.013 \mathrm{~km}$ so $v_{1}=1.6958 \mathrm{~km} / \mathrm{s}, v_{2}=1.6173 \mathrm{~km} / \mathrm{s}$.

\section{$B$ Determine the Position in the Perilune and Apolune}

- The establishment of coordinate system

In the landing point longitude circle plane, set the intersection between the line of month heart/trajectory perilune and the moon surface as the coordinate origin, the spacecraft perilune velocity direction is as $X$ axis, the moon heart directed to perilune direction as $y$ axis.

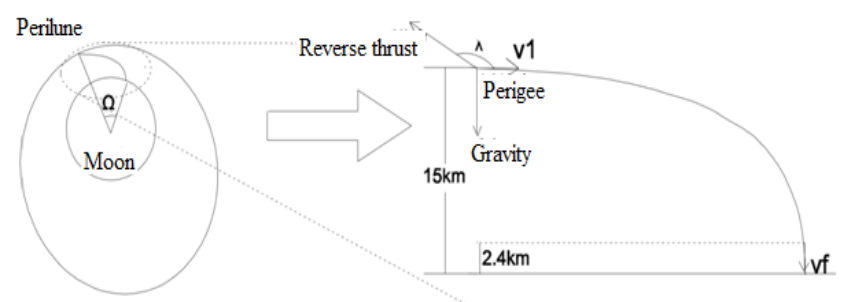

Figure 1. Schematic diagram of reference system

- Equivalent treatment of variable parameters

Because the reverse thrust in the main reducer stage is from the fuel consumption, the craft weight gradually reduces [5]. If the weight is seen as a function of time, $m_{t}=m_{0}-\int_{0}^{t} \dot{m} d t, \dot{m}$ is fuel consumption kilograms for unit time. As $m$ changes with time and is uncertain, it is difficult to obtain the numerical solution, this paper uses $m$ to replace spacecraft weight. And airship end weight 
is $\dot{m}_{f}=1400 \mathrm{~kg}, \bar{m}=\frac{m+m_{f}}{2}, \dot{m}=2400 \mathrm{~kg}$, so $\bar{m}=1900 \mathrm{~kg}$.

Reverse thrust is between the $1500 \mathrm{~N}$ and $7500 \mathrm{~N}$, its variation equation $F(t)$ is unknown. So we can consider changing unknown variables into a constant. $\bar{F}$ is average thrust within time $t$, then use the constant value $\vec{F}$ equivalent to reverse thrust. Before equivalent, the spacecraft momentum is $I=\int_{0}^{t} F(t) d t$; after equivalent, the spacecraft momentum is $I^{\prime}=\bar{F}^{*} t$. Reverse thrust equivalent principle is the spacecraft's momentum is constant equivalent before and after the equivalent.

$$
\int_{0}^{t} F(t) d t=\bar{F}^{*} t=I .
$$

The momentum value can be obtained according to the definition of momentum.

$$
I=\int F d t=\int v_{e} m d t=v_{e} \int m d t=v_{e}\left(m-m_{f}\right)
$$

While $v_{e}=2490 \mathrm{~m} / \mathrm{s}, m-m_{f}=1000 \mathrm{~kg}$. So the actual momentum is $I=249000 \mathrm{~N} * \mathrm{~s}$. So the formula can be obtained:

$$
\bar{F}^{*} t=294000 .
$$

The motion process is divided into horizontal and vertical stages. So the horizontal seed change process is:

$$
\frac{\bar{m} g_{\text {moon }}-\bar{F} \sin \lambda}{-} * t=v_{f} .
$$

The displacement change in the vertical direction is:

$$
\frac{\bar{m} g_{\text {moon }}-\bar{F} \sin \lambda}{2 \bar{m}} * t^{2}=x_{v} \text {. }
$$

$g_{\text {moon }}=1.633 \mathrm{~m} / \mathrm{s}^{2}$ is gravity acceleration in the moon's surface, $\lambda$ is the angle between the speed direction and $\stackrel{-}{F}, \quad v_{f}=57 \mathrm{~m} / \mathrm{s}$ is the vertical end speed, $x_{v}=1260 \mathrm{~m}$ is the vertical end displacement, $t$ is the motion time. And $\bar{F}, \lambda, t$ are the unknown parameters. From the formulas (5), (6) and (7), they can get $\bar{F}=6650 \mathrm{~N}, \lambda=154.55^{\circ}, t=442.11 \mathrm{~s}$.

- The specific perilune position
In the longitude circle plane of landing point, the position between Cartesian coordinates and the moon is as shown in Figure 2.

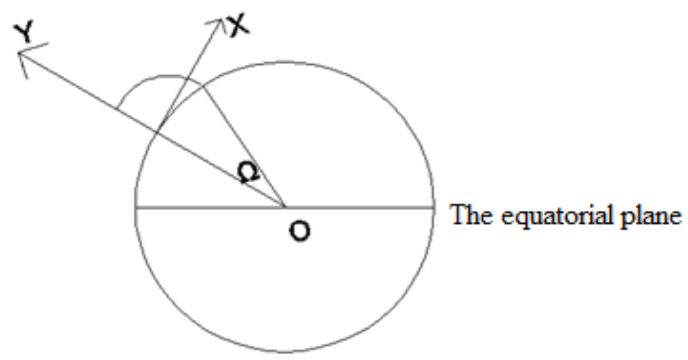

Figure 2. The relative position between Cartesian coordinates and the moon

Because the selenocentric angle is minimum, so the horizontal displacement of spacecraft is equal to arc length of central angle. So the displacement of the phase angle changes on heart and the $\mathrm{X}$ axis direction, $x_{h}=r \cdot \Omega$. Put the $\lambda, t$

into the horizontal motion equation $x_{h}=v_{1} t+\frac{\bar{F} \cos (\lambda) t^{2}}{2 \bar{m}}$, so the horizontal displacement is $x_{h}=440866.4 \mathrm{~m}$. Because the lunar radius is known, selenocentric angle is $\Omega=0.2538 \mathrm{rad}=14.54^{\circ}$.

Because the spacecraft perilune is in south of north latitude 44.12 degrees latitude coil, so perilune latitude is $29.58 \mathrm{~N}$, so perilune location is $(19.51 \mathrm{~W}, 29.58 \mathrm{~N}$, altitude $12.359 \mathrm{~km})$.

- The apolune position determination

Because apolune and perilune are at the long axis endpoint of elliptical orbit, and the moon bar center is at the focal point of ellipsoid, combined geometric relations with geography latitude and longitude knowledge, the apolune is (160.49E, 29.58S, altitude 97.359km).

\section{The Optimal Control Strategy In the Main DeCELERATE SeCtion}

\section{A The Discretization Main Deceleration Track}

In order to overcome the main thrust and direction change in the decelerate stage; this paper uses differential idea to do discretization on the moon soft landing orbit [6]. The time in main decelerate period can be divided into $n \Delta t$. This Infinitesimal will be divide time axis into $t_{0}, t_{1}, \cdots, t_{i-1}, \cdots, t_{n}$. For each infinitesimal stage, as the time interval is small enough, it can ignore thrust $F$ and $\theta$ direction change. Based on the discrete, each section can be done stress analysis, select a time infinitesimal $t_{i}$ to carry on the analysis of dynamic process.

Weight variation in the infinitesimal is: 


$$
\Delta m=\frac{F}{v_{e}} \Delta t .
$$

Weight relationship between adjacent times is:

$$
m_{l+\Delta t}=m_{t}-\Delta m \text {. }
$$

The horizontal acceleration is:

$$
a_{t x}=-\frac{F \cos (\theta)}{\left(m_{l}+m_{l+\Delta t}\right) / 2} .
$$

The horizontal speed relationship in adjacent time is:

$$
v_{t x+\Delta t}=v_{t x}+a_{l x} \Delta t .
$$

The vertical acceleration is:

$$
a_{t y}=g_{\text {moо }}-\frac{F \sin (\theta)}{\left(m_{l}+m_{l+\Delta t}\right) / 2} .
$$

The vertical velocity relationship in adjacent time is:

$$
v_{t y+\Delta t}=v_{t y}+a_{i x} \Delta t .
$$

The horizontal displacement is:

$$
x_{i}=\frac{v_{t x}+v_{t x+\Delta t}}{2} \Delta t .
$$

The vertical displacement is:

$$
y_{i}=\frac{v_{t y}+v_{t y+\Delta t}}{2} \Delta t .
$$

\section{B The Establishment of Single Objective Nonlinear \\ Programming Model based on the Recursive Relationship}

In the main deceleration stage, the airship is mainly controlled by acceptor engine. Here we will see the ship as a particle, only study the photosynthetic thrust $\bar{F}$ provided by all engines. In order to obtain the optimal control strategy,

we need to determine the $F$ and $\theta$ changing with time. First of all the motion process is discredited according to the above section.

Determine the target function: in the main deceleration stage it needs to consume large amounts of fuel. How to choose the appropriate control strategy to make the fuel consumption least is an urgent task at the constant fuel utilization rate [7]. So the process of the objective function is the maximum amount of fuel remaining:

$$
\max \left(m_{0}-\sum_{i=1}^{n} \Delta m_{i}\right) \text {. }
$$

Constraint conditions and the decision variables: the perigee is as the initial condition, $m_{0}=2400 \mathrm{~kg}, x_{0}=0, y_{0}=0, v_{x 0}=1700 \mathrm{~m} / \mathrm{s}, v_{y 0}=0$. According to the recursive process of formulas (9), (11), (13), (14) and (15), it can get the weight for each interval, horizontal speed, vertical velocity, horizontal displacement, vertical displacement.

The vertical displacement of main deceleration stage is $12000 \mathrm{~m}$, horizontal displacement is $440866.40 \mathrm{~m}$ (the first Q obtained), and the velocity of main deceleration segment end point is $57 \mathrm{~m} / \mathrm{s}$. So we can have the following equality constraints:

$$
\begin{aligned}
& \sum_{i=1}^{100} x_{i}=440866.4 \\
& \sum_{i=1}^{100} y_{i}=12000 \\
& \sqrt{v_{x m}^{2}+v_{y m}^{2}}=57
\end{aligned}
$$

\section{Using GENETIC AlgORITHM TO FInD THE GLOBAL} OPTIMAL SOLUTION

This paper uses genetic algorithm, the objective function $m_{0}-\sum_{i=1}^{n} \Delta m_{i}$ as fitness function and the three equations as constraint conditions to solve the maximum value of the objective function. As the existence of equality constraints, so this paper uses the penalty function method to process the constraint condition, and produces the new fitness function on the basis of original.

This paper directly uses the MATLAB genetic algorithm toolbox, set 600 populations, genetic algebra 200 to get genetic algorithm optimization. The interception of five consecutive operations results are show in Table 1 . As the genetic algorithm results have uncertainty, the data has some fluctuation.

TABLE 1. THE GENETIC ALGORITHM RESULTS

\begin{tabular}{|c|c|c|c|c|}
\hline $\begin{array}{c}\text { Weight } \\
\text { after } \\
\text { decelerate } \\
(\mathrm{t})\end{array}$ & $\begin{array}{c}\text { Speed } \\
\text { after } \\
\text { decelerate } \\
(\mathrm{m} / \mathrm{s})\end{array}$ & $\begin{array}{c}\text { The } \\
\text { falling } \\
\text { height } \\
(\mathrm{km})\end{array}$ & $\begin{array}{c}\text { Horizontal } \\
\text { displacement } \\
(\mathrm{km})\end{array}$ & $\begin{array}{c}\text { Decelerate } \\
\text { time }(\mathrm{s})\end{array}$ \\
\hline 1.29 & 45.69 & 12.30 & 450.71 & 470.00 \\
\hline 1.31 & 83.00 & 11.60 & 443.23 & 460.00 \\
\hline 1.29 & 50.84 & 11.94 & 448.19 & 470.00 \\
\hline
\end{tabular}

In Table 1, it can be seen that the operating results are different. We select the first running results as the final result.

\section{The Optimal Control Strategy at Obstacle} Avoidance Stage

The range of obstacle avoidance section is between $2.4 \mathrm{~km}$ and $30 \mathrm{~m} \mathrm{t}$ from the lunar surface, the main requirement is to avoid large craters and gravel, determine the fall place. We first use the aerial image to extract digital elevation map matrix, then do two values process, set the threshold to distinguish a pit and relatively flat ground.

First we extract digital elevation map matrix, and the matrix is changed into double type, and make gray distribution histogram as shown in Figure 3. 


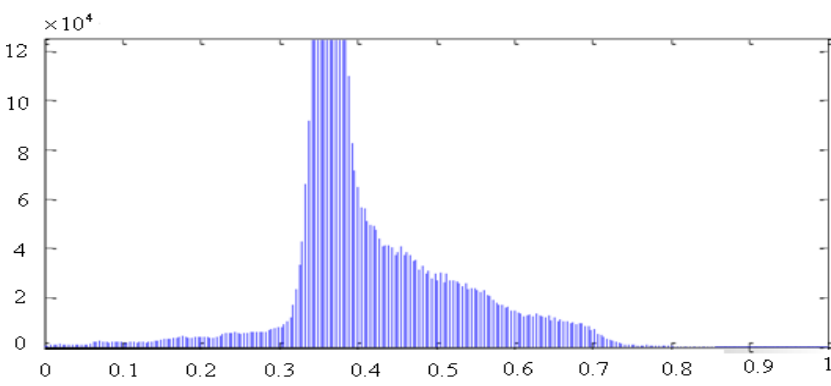

Figure 3. Gray Distribution histogram for coarse obstacle avoidance phase

The gray distribution is most concentrated between 0.333 and 0.4 , and the corresponding elevation range is from $85 \mathrm{~m}$ to $102 \mathrm{~m}$, which can be considered relatively flat. Set the gray value in the range as 1 , the rest is 0 , so the distribution map of greatly pit and flat place is obtained.

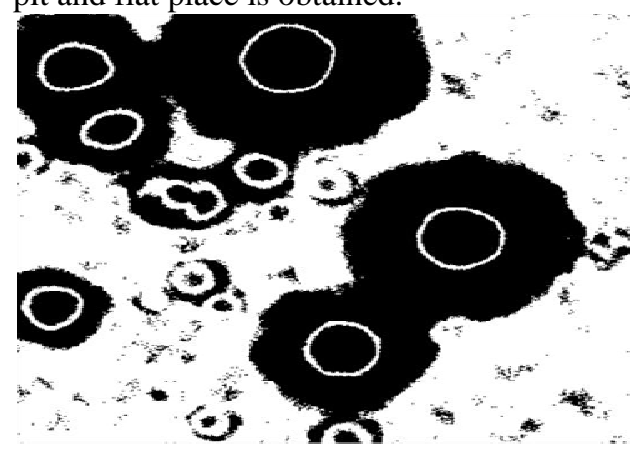

Figure 4. Coarse obstacle avoidance gray treatment plan

\section{A The Implementation of Obstacle Avoidance}

- Determine the landing point

If the height of any point in a square region $100 \mathrm{~m} * 100 \mathrm{~m}$ is $85 \sim 102 \mathrm{~m}$, the area is a flat area. With the center point of this square, find whether there is flat area, then calculate the distance between regional center point and spacecraft projection in lunar point, finding the shortest distance points, namely the initial landing place. Through calculation, it is at the 1091st row and 1091st column, according to the original image, it can be changed into coordinate $(1091,1209)$, and the distance between this point and spacecraft projection on the moon is $83.44 \mathrm{~m}$.

- Determine the spacecraft engine horizontal thrust in the transfer process

The spacecraft horizontal velocity is zero before and after the obstacle avoidance transfer. So the spacecraft accelerates in the horizontal direction, and then slows down to zero. The optimal control scheme is the pursuit of the minimum energy consumption, and uniformly accelerated motion can maximize the utilization rate of the fuel. So the spacecraft uniform accelerates at the horizontal direction, and then decelerates to zero.

$$
\frac{1}{2} a_{b}\left(\frac{t}{2}\right)^{2}=\frac{l}{2}
$$

$$
F_{c}=m_{c} a_{c} .
$$

From the formula (17), it can get $a_{b}=0.49 \mathrm{~m} / \mathrm{s}^{2}$. The weight can be ignored at the obstacle avoidance section relative to main deceleration section, so $m_{c}=m_{0}=1372.2 \mathrm{~kg}, F_{c}=672.38 \mathrm{~N}$. At the thrust direction: the first 13 seconds, the spacecraft projection point directs to the preliminary landing point; the second 13 seconds it directs to the spacecraft projection point.

\section{SUMMARY}

According to the law of energy conservation and the second law of Kepler, this paper obtains the spacecraft velocity in perilune and apolune. Based on trajectory inversion thought, this paper obtains position and velocity direction in the perilune and apolune. Then the paper establishing optimization model of genetic algorithm for the main deceleration section. Aiming at the obstacle avoidance section, based on the threshold selection, this paper uses image segmentation method to do two valued processing on gray matrix. According to the landing requirement, this paper uses MATLAB programming to obtain landing point coordinate and control scheme.

\section{REFERENCES}

[1] S.K. Si, X.J. Sun. Genetic algorithms. Beijing: National Defense Industry Press, 2011: 305-307.

[2] G. Chen, Z.M. Wan, M. Xu. Research on parameter and constraint handling method of aircraft trajectory optimization using genetic algorithm. Journal of system simulation, 2012, 17(11): 23-26.

[3] Alexander I. Kozynchenko. Enhancing the manoeuvring capabilities of a lunar landing module using predictive guidance algorithms. Acta Astronautica, 2011, 67(3): 406-416.

[4]S.K. Si, X.J. Sun. Soviet lunar sample return missions: Landing site identification and geologic context. Planetary and Space Science, 2012 69(1): 76-88.

[5] Alexander I. Kozynchenko. Study on passive momentum exchange landing gear using two-dimensional analysis. Acta Astronautica, 2014, 105 (2): 407-416.

[6] Jeremiah N. Mpagazehe, Kenneth W. Street Jr. An experimental study of lunar dust erosive wear potential using the JSC-1AF lunar dust simulant. Wear, 2014, 316 (1): 79-91.

[7] P. Weiss, K.L. Yung. Feasibility study of a lunar landing area navigation network deployed by impacting micro-probes. Planetary and Space Science, 2011, 58(6): 893-903. 